\title{
Patient education integrated with acupuncture for relief of cancer-related fatigue randomized controlled feasibility study
}

Michael F Johnston ${ }^{1,2,3}$, Ron D Hays ${ }^{2,3,4}$, Saskia K Subramanian ${ }^{5}$, Robert M Elashoff ${ }^{6,7}$, Eleanor K Axe ${ }^{1}$, Jie-Jia Li ${ }^{1}$, Irene Kim¹, Roberto B Vargas ${ }^{1,2,3,4}$, Jihey Lee ${ }^{6}$, LuGe Yang ${ }^{1}$ and Ka-Kit Hui ${ }^{1,2,3^{*}}$

\begin{abstract}
Background: Cancer-related fatigue (CRF) is a prominent clinical problem. There are calls for multi-modal interventions.

Methods: We assessed the feasibility of delivering patient education integrated with acupuncture for relief of CRF in a pilot randomized controlled trial (RCT) with breast cancer survivors using usual care as control. Social cognitive and integrative medicine theories guided integration of patient education with acupuncture into a coherent treatment protocol. The intervention consisted of two parts. First, patients were taught to improve self-care by optimizing exercise routines, improving nutrition, implementing some additional evidence-based cognitive behavioral techniques such as stress management in four weekly 50-minute sessions. Second, patients received eight weekly 50-minute acupuncture sessions. The pre-specified primary outcome, CRF, was assessed with the Brief Fatigue Inventory (BFI). Secondary outcomes included three dimensions of cognitive impairment assessed with the FACT-COGV2.
\end{abstract}

Results: Due to difficulties in recruitment, we tried several methods that led to the development of a tailored recruitment strategy: we enlisted oncologists into the core research team and recruited patients completing treatment from oncology waiting rooms. Compared to usual care control, the intervention was associated with a 2.38-point decline in fatigue as measured by the BFI (90\% Confidence Interval from 0.586 to 5.014; $p<0.10$ ). Outcomes associated with cognitive dysfunction were not statistically significant.

Conclusions: Patient education integrated with acupuncture had a very promising effect that warrants conducting a larger RCT to confirm findings. An effective recruitment strategy will be essential for the successful execution of a larger-scale trial.

Trial registrationNCT00646633

\section{Background}

Cancer-related fatigue (CRF) is an unrelenting sensation of tiredness far disproportionate to levels of physical activity. It is unmitigated by rest/sleep and is physically debilitating $[1,2]$. Patients with many types of cancer report CRF as their most severe symptom [3]. CRF compromises ability to complete daily routines (such as food preparation, household chores, and social activities) and

\footnotetext{
* Correspondence: khui@mednet.ucla.edu

'UCLA Center for East-West Medicine, David Geffen School of Medicine at UCLA, LA, USA

Full list of author information is available at the end of the article
}

impairs ability to attend/perform work [4-6]. CRF is also the most prevalent symptom $[3,4]$. In a systematic review, authors estimate CRF to range from $39-90+\%$ during treatment and $19-38 \%$ after treatment [7]. CRF is multi-factorial. It appears to result from various exogenous and endogenous factors that dysregulate physiological, biochemical, and psychological systems $[8,9]$.

Alleviating CRF is a high-priority issue, [10] with a documented need for new treatment approaches [11]. The National Comprehensive Cancer Network (NCCN) enumerates many pharmacologic and non-pharmacologic options [2]. A Cochrane [12] and associated review 
[11] of pharmacologic treatments for CRF finds positive results for only three: methylphindate (based on 2 small trials, one with non-significant results) and two haemopoetic growth factors: erythropoietin and darbopeitin $[11,12]$. The growth factors improved anemia-associated CRF, but recovery was often incomplete and was accompanied by potential side-effects $[11,12]$. Authors of a Cochrane review found exercise to provide some benefit, both during and after cancer treatment [13]; however authors of other reviews were less optimistic [14,15]. Addressing these discrepant interpretations, Stone and Minton [11] commented that many studies showed that exercise improved physical function without reducing fatigue. A Cochrane review [16] on psychosocial interventions during cancer treatment found limited promising evidence for several specific approaches. Though not enumerated in the NCCN recommendations, other research reports showed promising evidence from pilot studies for acupuncture [17-21] and acupressure [19,22].

Prior studies typically investigated treatment options using a single modality [23]. However, CRF often persists precisely because its complex etiology renders mono-modality approaches limited [23]. Accordingly, we drew upon elements of social cognitive theory [24] and integrative medicine theory [25-27] to create a four-session education program that involved teaching patients self-care techniques alongside an eight-session program that involved administering acupuncture. This integrated program addressed the call for new treatment options that are multimodal [28] and act in a broad-based manner [29].

This study assessed the feasibility of conducting a larger-scale randomized controlled trial (RCT) on the effectiveness of this integrated patient education and acupuncture program in relieving CRF. In doing so, we developed two specific objectives. First, to design a strategy for recruitment tailored to the institutional environment and the therapeutic intervention. This is important because successful recruitment strategies sometimes perform poorly in CAM-based research trials, [26,30-32] even though patients in the community are tremendously interested in CAM for symptom relief [33]. The second objective was to collect evidence of preliminary effects. This follows from the recommendation of a National Cancer Institute Community Clinical Oncology Program team that concluded small-scale studies are of critical importance for improving evidencebased assessments of CAM treatment regimens [34,35].

\section{Methods}

We report study randomization procedures following the statement on Consolidated Standards of Reporting Trials (CONSORT). We generated a random allocation sequence (1:1 ratio, permuted block randomization with blocks of size four) prior to recruiting subjects using the system found at http://www.randomization.com. The confidential randomization scheme was maintained in a computer isolated from the clinical team. Upon patient enrollment, the clinical team notified the study administrator who secured the sequence-appropriate assignment. This study was open (there was no blinding for patients, providers, or study investigators) and the randomization sequence remained concealed until study completion.

Trial inclusion/exclusion criteria were adopted from the earliest published study on acupuncture for CRF [18]. Inclusion criteria were that a patient is female, between 18 and 65, finished with primary therapy for breast cancer (free of cancer), and fatigued as measured by a score of 4 or greater on the Brief Fatigue Inventory (BFI) [18]. The exclusion criteria were depression (Hospital Anxiety and Depression Score [HADS] depression score $>10$ ), severe anemia at intake (Hemoglobin level $<9 \mathrm{~g} / \mathrm{dL}$; hematocrit level <30; a decline in hemoglobin of $2 \mathrm{~g} / \mathrm{dL}$ over the previous month; or in active treatment for anemia), or severe limitations in physical functioning (Kornofsky score $<70$ ). To maintain consistency with the inclusion/exclusion criteria from the earlier published study [18], we did not exclude patients with other potential causes of fatigue, such as hypothyroidism and hepatitis.

\section{Recruitment}

We intended to meet a recruitment goal of 80 (based on a published power analysis [17]) by enlisting nearly 80 subjects from a prior study of symptoms conducted by a team member (SKS). However, we soon realized the need to diversify recruitment methods and tried several nontailored recruitment methods. We ran newspaper ads, posted flyers in numerous clinics, cancer wellness centers, and support group meeting sites. We designed an oversized poster with formatted text/graphics and placed it in a heavily-trafficked hospital hallway. We posted an electronic announcement about the study in an email sent to approximately 700 institution-affiliated clinicians, researchers, and professors. Further, the study's Principal Investigator $(\mathrm{KKH})$ sent a personal letter to 14 physician colleagues in which he requested referrals.

With the hope of developing a strategy to overcome continued difficulties with recruitment, we consulted the Director of the UCLA Survey Research Center. The Director recommended that we extend our recruitment efforts to an oncology waiting room (T. Hays, personal communication, July, 2008). We then worked on logistics with a clinic manager and nurse practitioner of an oncology practice that regularly refers patients to the clinic that was providing the study treatment (the UCLA Center for East-West Medicine). The clinic manager and 
nurse practitioner sought the support of their clinic's oncologists. These efforts resulted in a tailored recruitment strategy that involved enlisting oncologists into the research project and sending a recruiter to the oncology waiting room at predetermined times to enroll patients who were completing treatment.

\section{Intervention}

A growing body of evidence suggests that interventions that are informed by theory, especially those combining multiple theories, are more effective than interventions that lack a theoretical basis [24]. A multidisciplinary team of medical researchers, acupuncturists, and physicians knowledgeable about patient education and acupuncture (MFJ, EKA, JJL, IK, KKH) developed our study's therapeutic protocol with reference to two bodies of theory: Social Cognitive Theory and Integrative Medicine. The team used Social Cognitive Theory to conceptualize and enhance self-efficacy, [36] which is defined as a person's confidence in her ability to perform a specific behavior [37]. Theorists consider self-efficacy to be the key catalyst of behavioral change [24]. Practitioners may use four distinct modes (ways) to help patients develop self-efficacy $[36,38,39]$. Additional file 1 draws upon Social Cognitive Theory literature $[24,36-38,40]$ to communicate various ways in which IK, the study clinician who delivered patient education, sought to improve self-efficacy in patients. The "Assessment" column identifies mechanisms that IK used to assess patient adherence to self-care recommendations. The final column ("Mode of facilitating self-efficacy") provides a step-by-step accounting of the system the team developed to increase IK's fidelity to the self-care protocol across patients. In short, IK used the protocol to work with patients to develop individualized achievable goals accompanied by feasible tasks. This approach was designed to help patients more thoroughly implement recommendations; it has also been reported that this approach helps improve patients' confidence [39].

Our team used Integrative Medicine theory $[26,27,41-43]$ to blend several non-pharmacologic options into a coherent protocol oriented towards addressing CRF in the context of healing the whole person. The team set, as a high priority, the development of a strong, positive clinician-patient bond by arranging for study acupuncturists to conduct in-depth, personcentered clinical intakes. During intake, CRF was treated as a signal pointing to clues for developing a wellness plan. Across all treatment sessions the study acupuncturists sought to help patients improve their self-care by tailoring advice according to TCM diagnosis, engaging in dialogue through presenting common ideas about wellbeing with a TCM-based perspective, and refocusing away from a quick fix mentality to a longer-term holistic perspective [26]. Conventionally trained oncologists, physicians and researchers provided substantial input as the team developed the treatment protocol.

The first four sessions of the therapeutic protocol involved IK administering self-care training alongside acupuncture. For the four weekly self-care sessions, IK sought to help patients enhance their general well-being through facilitating lifestyle changes in the areas of self-massage, exercise, nutrition, and stress management (relaxation) (Additional file 1). She also provided fatigue-specific recommendations in the form of dietary advice (Additional file 1). A second acupuncturist (JJL) provided the eight weekly acupuncture sessions. These were individualized according to one of four different symptom patterns of dysfunction that could trigger CRF and other problems. He also needled four energy-associated points (Table 1). The team anticipated that patients would require several weeks to incorporate effective self-care. Believing that acupuncture would produce more immediate benefits, the team provided education during the first four weeks of treatment rather than in the middle or at the end of the eight week therapeutic protocol.

In brief, a multidisciplinary team utilized Social Cognitive Theory and Integrative Medicine Theory to develop a therapeutic protocol that would address fatigue in the context of caring for the whole person. To do so, the practitioners oriented patients towards a holistic view of health, built up their self-efficacy through a structured set of progressively challenging mastery experiences, and delivered acupuncture early on to achieve results that would enhance commitment to and confidence in selfcare.

\section{Usual Care}

Participants in both arms of the study received usual care from their personal physicians according to the principles of normal practice which included pharmacologic and non-pharmacologic options [2].

\section{Outcome}

We pre-specified the study's primary outcome to be CRF as measured by the BFI at follow-up. The BFI is a scale composed of 9 items that assess intensity of present, usual, and worst fatigue during past 24 hours and interference with usual activities [18]. Response choices range from 0 (no fatigue) to 10 (worst imaginable fatigue) [18]. A single score ranging from 0 to 10 was computed for the BFI by averaging responses to all 9 items. Previous research provided evidence in support of the reliability and validity of the BFI [18]. Cut-off scores distinguish mild (1-3), moderate (4-6), and severe (7-10) CRF that correspond to the NCCN-recommendations for the CRF clinical practice guidelines screening question: "How would you rate your fatigue on a scale of 0-10 over the past 7 days?" [2]. 
Table 1 Extract from Acupuncture Protocol, presented in accordance with STandards for Reporting Interventions in Controlled Trials of Acupuncture (STRICTA) ${ }^{68}$

\begin{tabular}{|c|c|c|c|}
\hline Item & Domain & Item description & Study-specific details \\
\hline 1 & $\begin{array}{l}\text { Acupuncture } \\
\text { rationale }\end{array}$ & $\begin{array}{l}\text { Rationale for selection of points } \\
\text { and literature sources }\end{array}$ & The acupuncturist needled four energy-associated acupuncture points on all patients. ${ }^{69}$ \\
\hline 2 & $\begin{array}{l}\text { Acupuncture } \\
\text { rationale }\end{array}$ & $\begin{array}{l}\text { Individualization and literature } \\
\text { sources }\end{array}$ & $\begin{array}{l}\text { Based on clinical experience and TCM theory, }{ }^{69} \text { the study acupuncturist [name] } \\
\text { developed a system of classifying patients into one of four symptom pattern subgroups } \\
\text { that may contribute to the development of fatigue symptoms: (A) gastrointestinal } \\
\text { symptoms and signs, (B) emotional symptoms and signs, (C) sleep dysfunction symptoms } \\
\text { and signs, and (D) myofascial muscle pain. Classification took account of patient's } \\
\text { physiological dysfunctions as recognized by current state of health, previous disease } \\
\text { conditions, and treatment history. }\end{array}$ \\
\hline 3 & $\begin{array}{l}\text { Needling } \\
\text { details }\end{array}$ & Points used & $\begin{array}{l}\text { For each patient, the study acupuncturist administered acupuncture at } 4 \text { energy- } \\
\text { associated points (Li4, Sp6, ST36, Ki3) and then additionally needled acupuncture points } \\
\text { he considered beneficial primarily in light of symptom pattern classification. Those in the } \\
\text { gastrointestinal symptom pattern also received acupuncture on P6 and Sp4. Those in the } \\
\text { emotional symptom pattern also received acupuncture on Lu7, Ki4, Liver3, Yintang, and } \\
\text { GV20. Those in the sleep symptom pattern also received acupuncture on H7, Ki4, and } \\
\text { Ub62. Those in the pain symptom pattern also received acupuncture on Gb20, Te5, Gb43, } \\
\text { SI3, Ub62, Gb29, Gb30, Gb40, and selected points related to specific sites of somatic pain. } \\
\text { All points were administered bilaterally. }\end{array}$ \\
\hline 4 & $\begin{array}{l}\text { Needling } \\
\text { details }\end{array}$ & Depths of insertion & $\begin{array}{l}\text { Points were needled perpendicularly to a depth of } 0.5-1.5 \text {, with exact depth varying by } \\
\text { location of acupuncture point, ease with which patient achieved the "de qi" ("twitch") } \\
\text { sensation, patients' size, sensitivity, and their state of health. }\end{array}$ \\
\hline 5 & $\begin{array}{l}\text { Needling } \\
\text { details }\end{array}$ & Responses elicited & $\begin{array}{l}\text { The study acupuncturist sought to achieve the "de qi" (twitch) response by employing } \\
\text { needling techniques such as lifting, thrusting, and rotating. }\end{array}$ \\
\hline 6 & $\begin{array}{l}\text { Needling } \\
\text { details }\end{array}$ & Needle retention time & $\begin{array}{l}\text { Needles were inserted over the course of approximately } 10 \text { minutes, retained in place for } \\
\text { about } 30 \text { minutes, and removed over the course of about } 10 \text { minutes ( } 50 \text { minutes total) }\end{array}$ \\
\hline 7 & $\begin{array}{l}\text { Needling } \\
\text { details }\end{array}$ & Type of needles & $\begin{array}{l}\text { LEKON }^{\mathrm{TM}} \text { sterile disposable acupuncture needles of the following sizes: } 34 \mathrm{G} \text { (gauge) } \times 1.5 \\
(0.22 \mathrm{~mm} \times 40 \mathrm{~mm} \text { diameter); } 34 \mathrm{G} \times 1.0(0.22 \mathrm{~mm} \times 25 \mathrm{~mm}) ; 32 \mathrm{G} \times 1.5(0.25 \mathrm{~mm} \times 40 \\
\mathrm{mm}) ; \text { and } 38 \mathrm{G} \times 0.5(0.18 \mathrm{~mm} \times 13 \mathrm{~mm}) \text {; needle types were chosen according to } \\
\text { acupuncture point location and patients' size, sensitivity, and state of health. }\end{array}$ \\
\hline 8 & $\begin{array}{l}\text { Practitioner } \\
\text { background }\end{array}$ & Duration of relevant training & $\begin{array}{l}\text { The clinician delivering acupuncture [Jie-Jia Li] earned a medical degree from the } \\
\text { Southern Medical University in Guangzhou, China, a Master's degree in Traditional Chinese } \\
\text { Medicine from Shanghai University of Traditional Chinese Medicine, and a Ph.D. in } \\
\text { Traditional Chinese Medicine at American Liberty University in California. }\end{array}$ \\
\hline 9 & $\begin{array}{l}\text { Practitioner } \\
\text { background }\end{array}$ & Length of clinical experience & $\begin{array}{l}J \mathrm{JL} \text { has over } 20 \text { years of experience in providing acupuncture and has worked at the } \\
\text { UCLA Center for East-West Medicine for over } 15 \text { years. }\end{array}$ \\
\hline 10 & $\begin{array}{l}\text { Practitioner } \\
\text { background }\end{array}$ & Expertise in specific condition & $\begin{array}{l}\text { JJL has been treating patients with breast and other types of cancer for nearly } 10 \text { years; } \\
\text { he has been collaborating with [Name] in researching breast cancer for more than five } \\
\text { years. }\end{array}$ \\
\hline
\end{tabular}

To assess changes in cognitive complaints between first and last visit, we utilized the FACT-COG. This 50 item measure has 3 summary scores: cognitive problems, impact on functioning, and impact on healthrelated quality of life [44].

\section{Statistical Methods}

We used an analysis of covariance (ANCOVA) model. We fit ANCOVA with Huber's M estimation, a robust regression methodology [45]. The regression equation models BFI score at follow-up as a function of an intercept and two variables: BFI score at baseline and treatment status ( 0 if patient randomized to control and 1 if to treatment). Following published recommendations on statistical analysis for Phase II trials, $[46,47]$ we used a statistical significance criterion of $\mathrm{p}<0.10$ for a two-sided test on the primary outcome variable. One participant was randomized to usual care treatment but received patient education and acupuncture. She experienced a favorable response. Adhering to the randomization protocol, we kept her in the usual care group.

\section{Human Subjects}

Both the general and cancer-specific Institutional Review Boards at UCLA approved this study (\#06-010-01, \#POP0504401). All participants provided written informed consent.

\section{Results}

\section{Patient Recruitment}

Over 15 months, 40 people contacted the recruitment manager concerning the study. Figure 1 details the flow of participants through the recruitment, randomization, and treatment processes. Thirteen candidates were 


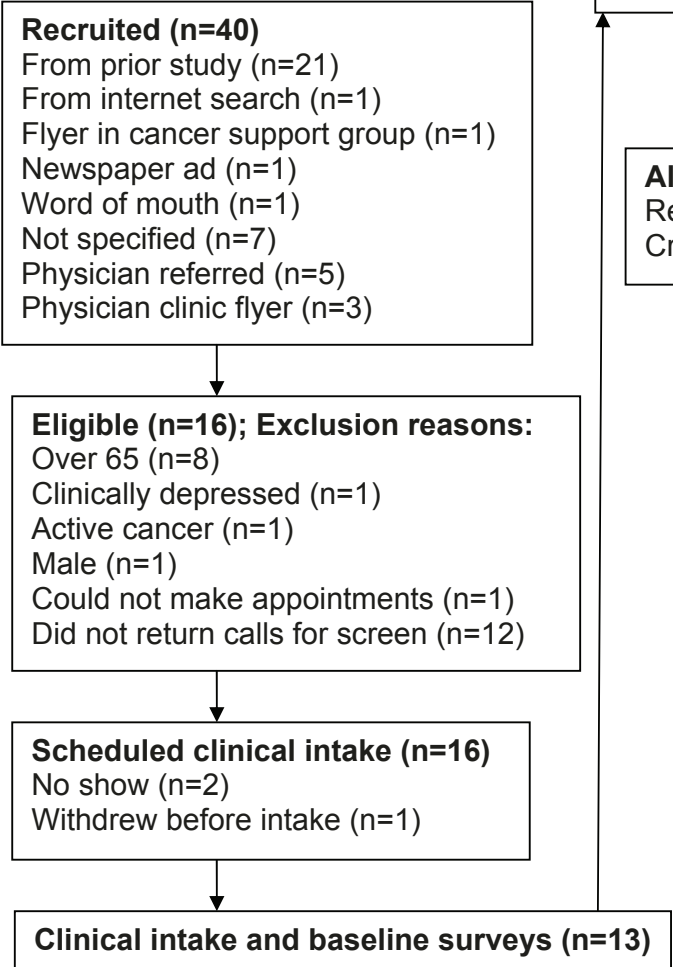

Figure 1 CONSORT diagram.

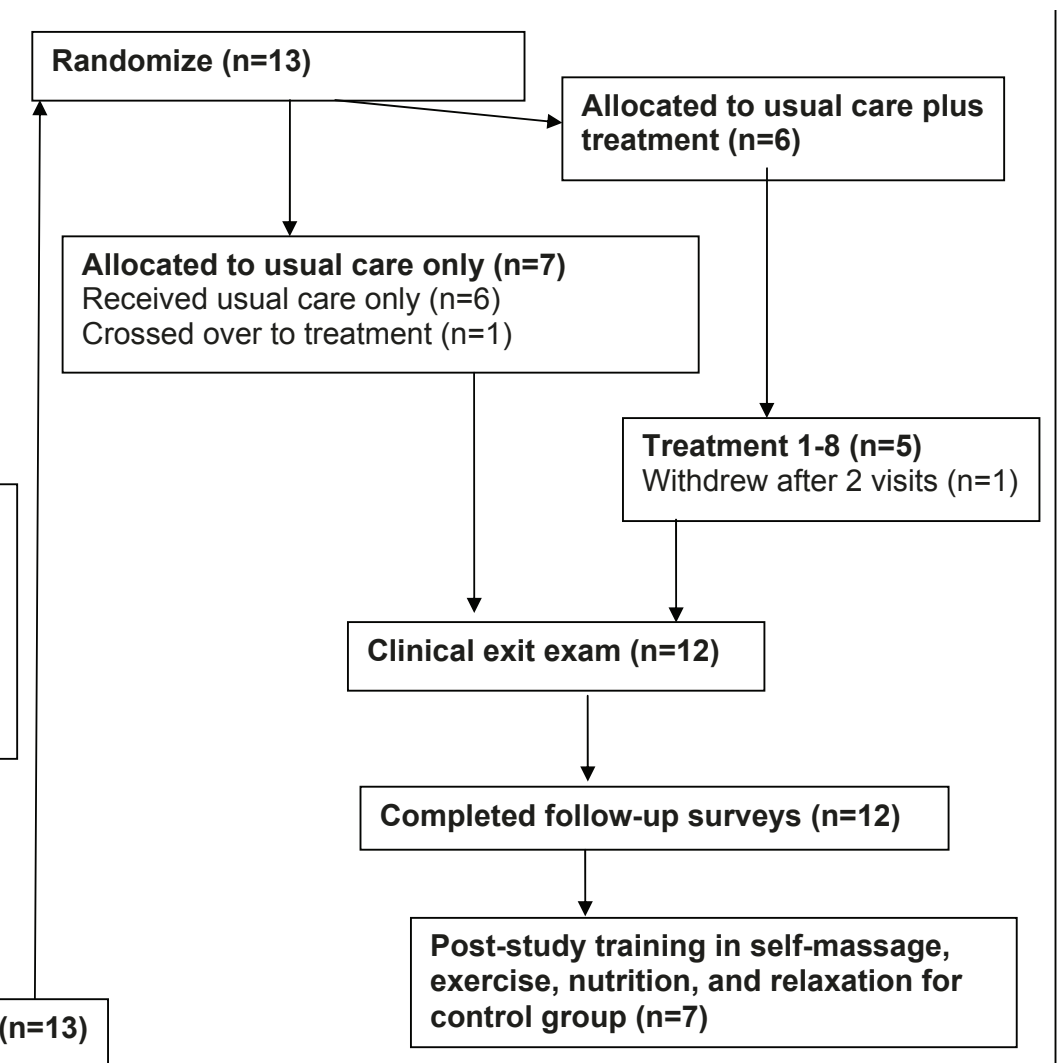

eligible; seven were randomized to control and six to treatment. One person in treatment withdrew after two weeks (no reason given). We were unable to collect any further information from this subject.

We began recruiting subjects by mailing the 80 women who had participated in an earlier open-ended interview study a letter inviting participation. Over approximately three months, 21 indicated a willingness to join. Of these, 8 were eligible and 5 participated in the research trial.

Responses to the initial (non-tailored) recruitment methods were limited. One woman responded to the newspaper advertisements. Three women contacted the recruitment coordinator after reading posted flyers. We had no indication that either the oversized poster or departmental email resulted in participants. The principal investigator's personalized letters resulted in 5 subjects contacting the recruitment manager. In sum, the non-tailored recruitment these 5 methods led approximately 9 people to express interest in the study over the course of 12 months.

In contrast, the tailored recruitment strategy was much more fruitful. Over the course of two weeks, a recruiter conducted two two-hour recruitment sessions that resulted in five patients expressing interest in our study. Extrapolated to a 12 month period, the tailored recruitment strategy may identify more than 100 breast cancer survivors who are interested in participating.

\section{Outcome Results}

Baseline demographic and clinical characteristics of the 12 study participants are shown in Table 2 . There were no statistically significant differences between the treatment $(n=5)$ and control $(n=7)$ subjects in terms of sociodemographic and clinical indicators (independent group t-tests). This indicates that the randomization procedure did not fail to provide for a fair comparison between treatment and control. Overall, the mean age of study participants was about 54 and about two-thirds of the participants were white.

The mean (standard deviation) scores of CRF, for the pre-specified primary outcome, CRF as measured by the BFI, were 6.33 (1.39) and 6.00 (1.09) at baseline for the treatment and control groups, respectively (See Table 3). This is close to a score of 7 which is considered "severe" fatigue. After the ten-week treatment period, the respective scores were 2.13 (1.23) and 4.38 (2.53). These correspond to "mild" and "moderate" fatigue in the treatment and control groups, respectively. The regression model 
Table 2 Participant baseline characteristics

\begin{tabular}{|c|c|c|c|}
\hline \multirow[b]{2}{*}{ Characteristic } & \multicolumn{3}{|c|}{ Number of Patients } \\
\hline & $\begin{array}{r}\text { Treatment } \\
\text { group } \\
(\mathrm{n}=5)\end{array}$ & $\begin{array}{r}\text { Control } \\
\text { group } \\
(\mathrm{n}=7)\end{array}$ & $\begin{array}{r}p \text {-value } \\
\text { (two-tailed) }\end{array}$ \\
\hline
\end{tabular}

$\begin{array}{lccc}\text { BFI } & 06.33(1.39) & 06.00(1.09) & 0.65 \\ \text { Age, years } & 55.00(6.40) & 53.00(7.20) & 0.58 \\ \text { Race } & & & 0.58 \\ \quad \text { White } & 4 & 4 & \\ \quad \begin{array}{l}\text { Black or African } \\ \text { American }\end{array} & 1 & 3 & \end{array}$

Income

$<\$ 20,000$

$\$ 20,000$ or higher

$\begin{array}{lll}0 & 2 \\ 5 & 5\end{array}$

Marital status

Married or

cohabiting

Single/divorced/

separated

Employment status

Employed

Unemployed

Chemotherapy

Yes

No

Radiation therapy

Yes

No

Hormone therapy

Yes

No

Severe pain

Yes

No

0.47

2

5

Note: p-values are from independent group t-tests.

estimates indicate that the treatment group patients benefited from a 2.38-point greater decline in CRF compared to the control group (See Table 4). The standard error of the treatment variable was 1.35 and the p-value 0.08 . Thus, the study provides evidence in favor of a treatment benefit.

A reduction in our pre-specified primary endpoint CRF as measured by the BFI is suggestive of a clinically meaningful outcome. Based on the baseline pooled standard deviation and post-treatment means, the effect size was 1.85. According to the Cohen's D metric, a large effect size is equal to or greater than 0.80 and a clinically meaningful difference is considered to be 0.20 or greater [48].

The evidence failed to suggest that the treatment decreased cognitive complaints.

The clinician performing acupuncture (JJL) was provided with a standardized checklist to assess for adverse events after each treatment, including bruising at needling site, panic, severe disorientation, fainting, infection, or puncture of an internal organ. The completed reports indicated that the acupuncture treatments were not associated with any adverse events.

\section{Discussion}

Responding to requests from the oncology community for a multimodal and broad-acting therapy to address CRF, $[7,8]$ we drew upon social cognitive theory and integrative medicine theory to assist patients with improving self-care, especially in terms of exercise and nutrition, in the context of providing acupuncture. The hypothesis that integrating patient self-care into treatment routines enhances clinical outcomes rests upon mixed findings [49]. One possible explanation for our positive results resides in the growing body of evidence suggesting that interventions informed by theory, especially those combining multiple theories, are more effective than interventions lacking a theoretical basis [24].

Participants in the treatment group experienced a 66\% reduction in fatigue (see Table 4: (6.33-2.13) / 6.33). This result compares very favorably with similar studies examining acupuncture or acupressure in the relief of CRF [18-22]. We believe that our treatment protocol integrating patient education with acupuncture provides more benefit than acupuncture alone because our protocol enhances self-care and effective self-care produces substantial positive health benefits.

\section{Limitations}

The foremost limitation of this study is the small sample size. We began the study anticipating that we would be able to recruit nearly 80 subjects from a previous study of symptoms in 80 breast cancer survivors. While succeeding in recruiting only 5 participants, we realized that such a strategy would not be dependable for future studies. Recognizing the need to diversify our recruitment methods, we tried several additional non-tailored approaches. In this process, we realized there are at least two distinct types of breast cancer survivors, each of which is reachable in very different ways. The first is patients finishing primary treatment and transitioning from the care of an oncologist to a primary care 
Table 3 Descriptive statistics

\begin{tabular}{|c|c|c|c|c|}
\hline \multirow[t]{2}{*}{ Variable } & \multicolumn{2}{|c|}{ Treatment $(n=5)$} & \multicolumn{2}{|c|}{ Control $(n=7)$} \\
\hline & Baseline mean (SD) & Follow-up mean (SD) & Baseline mean (SD) & Follow-up mean (SD) \\
\hline \multicolumn{5}{|l|}{ Pre-specified primary outcome } \\
\hline $\mathrm{BFI}$ & $\begin{array}{l}06.33 \\
(1.39)\end{array}$ & $\begin{array}{l}02.13 \\
(1.23)\end{array}$ & $\begin{array}{l}06.00 \\
(1.09)\end{array}$ & $\begin{array}{l}04.38 \\
(2.53)\end{array}$ \\
\hline \multicolumn{5}{|l|}{ Secondary outcomes (FACT-COG) } \\
\hline Perceived cog impairments, QOL impact & $\begin{array}{l}02.70 \\
(1.24)\end{array}$ & $\begin{array}{l}03.55 \\
(0.21)\end{array}$ & $\begin{array}{l}01.58 \\
(1.27)\end{array}$ & $\begin{array}{l}02.21 \\
(1.22)\end{array}$ \\
\hline Perceived cognitive impairments & $\begin{array}{l}03.00 \\
(0.36)\end{array}$ & $\begin{array}{l}03.34 \\
(0.06)\end{array}$ & $\begin{array}{l}02.02 \\
(0.83)\end{array}$ & $\begin{array}{l}02.27 \\
(0.95)\end{array}$ \\
\hline Perceived cognitive abilities & $\begin{array}{l}03.23 \\
(0.54)\end{array}$ & $\begin{array}{l}03.51 \\
(0.49)\end{array}$ & $\begin{array}{l}01.86 \\
(0.71)\end{array}$ & $\begin{array}{l}02.33 \\
(0.84)\end{array}$ \\
\hline
\end{tabular}

Table 4 ANCOVA estimation of pre-specified primary, post-specified secondary, and ancillary outcomes

\begin{tabular}{llll}
\hline Outcome Variables & Treatment effect estimate & Standard error & P-value \\
\hline Pre-specified primary outcome & & & 01.35 \\
\hline Brief Fatigue Inventory (BFI) & -02.38 & & \\
& & & \\
Secondary outcomes & & 0.0776 \\
\hline Perceived cognitive impairments, QOL impact(FACT-COG) & -00.44 & 0.56 & 0.4286 \\
Perceived cognitive impairments (FACT-COG) & -00.16 & 0.30 & 0.6024 \\
Perceived cognitive abilities (FACT-COG) & -00.02 & 00.45 & 0.9609 \\
\hline
\end{tabular}

ANCOVA is fit with Huber's $M$ estimation, a robust regression methodology. ${ }^{44}$ Rregression equations model score at follow-up as a function of an intercept, pretreatment score and treatment (an indicator variable).

physician. The second is patients who have completely finished treatment and are seen solely by primary care physicians. The first could be identified at oncology clinics as they finish treatment while the second is a more geographically dispersed population requiring contact at a variety of recruitment sites including primary care clinics, cancer survivor group meetings, cancer survivor events, etc. We see recruitment of patients finishing treatment as much more feasible. Accordingly, we recommend that researchers interested in replicating our protocol focus on addressing CRF at the completion of active cancer treatment and invite oncologists to play a key role in study design, implementation, and result dissemination. In any case, thoughtful and proactive recruitment methods are needed for successful conduct of a larger trial.

There were several other limitations to our study. Our exclusion criteria were consistent with that of one study [18], but not others [e.g. [22]]. The self-care components of the protocol could have been more effectively monitored with standardized questionnaires that assessed changes in patient behaviors and responses to these changes. We did not examine any biological mechanisms through which the intervention might decrease CRF. Finally, the open (non-blinded) study design may have increased intervention efficacy

\section{Conclusion}

Our study has provided evidence to support the feasibility of conducting a larger-scale RCT on patient education integrated with acupuncture to reduce CRF.

\section{Additional material}

Additional file 1: CONSORT Diagram. A word document with a CONSORT diagram.

\section{Acknowledgements}

The Susan G. Komen Breast Cancer Foundation provided this project's primary funding (Award Number POP0504401 to Hui). Other organizations that provided financial support for this project include: the California Breast Cancer Research Program of the University of California (Grant Number 10FB-0049; Hui, Johnston); the Gerald Oppenheimer Family Foundation (Hui), and the Annenberg Foundation (Hui). Hays was also supported in part by the UCLA Resource Center for Minority Aging Research/Center for Health Improvement in Minority Elderly (RCMAR/CHIME; NIH/NIA Grant Award Number P30AG021684), the UCLA/ Drew Project EXPORT (NCMHD, 2P20MD000182), and the UCLA Older Americans Independence Center (NIH/ NIA Grant P30-AG028748). We thank Dr. Susan Ettner for commenting on a draft of the manuscript and Ms. Sue Fan for coordinating team efforts. An earlier version of this paper was presented as a poster at the UCLA Collaborative Centers for Integrative Medicine Conference on Integrative Medicine at UCLA on Wednesday, February 3, 2010.

\section{Author details}

${ }^{1}$ UCLA Center for East-West Medicine, David Geffen School of Medicine at UCLA, LA, USA. ${ }^{2}$ Division of General Internal Medicine, David Geffen School 
of Medicine at UCLA, LA, USA. ${ }^{3}$ Department of Health Services, UCLA School of Public Health, LA, USA. ${ }^{4}$ RAND, Santa Monica, CA, USA. ${ }^{5}$ Department of Psychiatry and Biobehavioral Sciences, David Geffen School of Medicine at UCLA, LA, USA. ${ }^{6}$ Department of Biostatistics, School of Public Health, UCLA, LA, USA. ${ }^{7}$ Department of Biomathematics, UCLA Graduate Division, LA, USA.

\section{Authors' contributions}

MJ helped conceive the study, helped perform the statistical analysis, and drafted the manuscript. RDH helped conceive the study, helped perform the statistical analysis, and edited the manuscript. SKS helped conceive the study, helped recruit participants, drafted the section on recruitment, and edited other parts of the manuscript. RME directed the statistical analyses and edited the manuscript. EKA helped conceive the study, performed the clinical intakes and physical examinations, and edited the manuscript. JJL treated patients, participated in drafting the results section, and reviewed the manuscript. IK treated patients, participated in drafting the results section, and reviewed the manuscript. RBV participated in coordinating the study and edited the manuscript. JL conducted statistical analyses, drafted part of the results section, and reviewed the manuscript. LGY carried out data entry, organized the bibliography, and reviewed the manuscript. As Principal Investigator, KKH directed and orchestrated the project, and this manuscript, from conception to conclusion. All authors read and approved the final manuscript.

\section{Competing interests}

The authors declare that they have no competing interests.

Received: 20 October 2010 Accepted: 25 June 2011

Published: 25 June 2011

\section{References}

1. Escalante CP, Manzullo EF: Cancer-related fatigue: the approach and treatment. J Gen Intern Med 2009, Suppl 2:S412-6.

2. National Comprehensive Cancer Network (NCCN) clinical practice guidelines in oncology ${ }^{\mathbb{B}}$. Cancer-related fatigue. Version 1.2010, @ 2007 National Comprehensive Cancer Network, Inc:[http://www.nccn.org], 2/5/2010 Update.

3. Hickok JT, Morrow GR, Roscoe JA, et al: Occurrence, severity, and longitudinal course of twelve common symptoms in 1129 consecutive patients during radiotherapy for cancer. J Pain Symptom Manage 2005, 5: 433-42, Nov;30.

4. Hoffman $\mathrm{M}$, Ryan $J \mathrm{~L}$, Figueroa-Moseley $\mathrm{CD}$, et al: Cancer-related fatigue: the scale of the problem. Oncologist 2007, Suppl 1: 4-10.

5. Curt GA, Breitbart W, Cella D, et al: Impact of cancer-related fatigue on the lives of patients: new findings from the Fatigue Coalition. Oncologist 2000, 5(5):353-60.

6. Groenvold M, Petersen MA, Idler E, et al: Psychological distress and fatigue predicted recurrence and survival in primary breast cancer patients. Breast Cancer Res Treat 2007, , 2: 209-19, Oct;105.

7. Prue G, Rankin J, Allen J, et al: Cancer-related fatigue: A critical appraisal. Eur J Cancer 2006, , 7: 846-63, May;42.

8. Ryan JL, Carroll JK, Ryan EP, et al: Mechanisms of cancer-related fatigue. Oncologist 2007, , Suppl 1: 22-34.

9. Wang XS: Pathophysiology of cancer-related fatigue. Clin J Oncol Nurs 2008, , 5 Suppl: 11-20.

10. Borneman T, Piper BF, Sun VC, et al: Implementing the Fatigue Guidelines at one NCCN member institution: process and outcomes. J Natl Compr Canc Netw 2007, 10: 1092-101, Nov;5.

11. Stone PC, Minton O: Cancer-related fatigue. Eur J Cancer 2008, , 8: 1097-104, May;44.

12. Minton O, Richardson A, Sharpe $M$, et al: A systematic review and metaanalysis of the pharmacological treatment of cancer-related fatigue. J Natl Cancer Inst 2008, , 16: 1155-66, Aug 20;100.

13. Cramp F, Daniel J: Exercise for the management of cancer-related fatigue in adults. Cochrane Database Syst Rev 2008, , 2: CD006145, Apr 16.

14. Jacobsen PB, Donovan KA, Vadaparampil ST, et al: Systematic review and meta-analysis of psychological and activity-based interventions for cancer-related fatigue. Health Psychol 2007, , 6: 660-7, Nov;26.

15. Knols $R$, Aaronson NK, Uebelhart $D$, et al: Physical exercise in cancer patients during and after medical treatment: a systematic review of randomized and controlled clinical trials. J Clin Oncol 2005, , 16: 3830-42, Jun 1;23.
16. Goedendorp MM, Gielissen MF, Verhagen CA, et al: Psychosocial interventions for reducing fatigue during cancer treatment in adults. Cochrane Database Syst Rev 2009, 1: CD006953, Jan 21.

17. Johnston MF, Hays RD, Hui KK: Evidence-based effect size estimation: an illustration using the case of acupuncture for cancer-related fatigue. BMC Complement Altern Med 2009, 1, Jan 13;9.

18. Vickers AJ, Straus DJ, Fearon B, et al: Acupuncture for postchemotherapy fatigue: a phase II study. J Clin Oncol 2004, , 9: 1731-5, May 1;22.

19. Molassiotis A, Sylt P, Diggins $H$ : The management of cancer-related fatigue after chemotherapy with acupuncture and acupressure: a randomised controlled trial. Complement Ther Med 2007, , 4: 228-37, Dec;15.

20. Mao JJ, Styles T, Cheville A, et al: Acupuncture for nonpalliative radiation therapy-related fatigue: feasibility study. J Soc Integr Oncol 2009, , 2: 52-8, Spring;7.

21. Balk J, Day R, Rosenzweig M, et al: Pilot, randomized, modified, doubleblind, placebo-controlled trial of acupuncture for cancer-related fatigue. J Soc Integr Oncol 2009, 1: 4-11, Winter;7.

22. Zick SM, Alrawi S, Merel G, et al: Relaxation acupressure reduces persistent cancer-related fatigue. Evid Based Complement Alternat Med 2011, 2011. pii: 142913

23. Mustian KM, Morrow GR, Carroll JK, et al: Integrative nonpharmacologic behavioral interventions for the management of cancer-related fatigue. Oncologist 2007, , Suppl 1: 52-67.

24. Glanz K, Bishop DB: The Role of Behavioral Science Theory in the Development and Implementation of Public Health Interventions. Annu Rev Public Health 2010.

25. Bell IR, Caspi O, Schwartz GE, et al: Integrative medicine and systemic outcomes research: issues in the emergence of a new model for primary health care. Arch Intern Med 2002, 2: 133-40, Jan 28;162.

26. Deng G, Weber W, Sood A, et al: Integrative Medicine Research: Context and Priorities. Paper commissioned for the Institute of Medicine Summit on Integrative Medicine and the Health of the Public. 2009, 1-51.

27. MacPherson H, Thomas K: Self-help advice as a process integral to traditional acupuncture care: implications for trial design. Complement Ther Med 2008, , 2: 101-6, Apr;16.

28. Kangas M, Bovbjerg DH, Montgomery GH: Cancer-related fatigue: a systematic and meta-analytic review of non-pharmacological therapies for cancer patients. Psychol Bull 2008, , 5: 700-41, Sep;134.

29. Yates $P$, Aranda $S$, Hargraves $M$, et al: Randomized controlled trial of an educational intervention for managing fatigue in women receiving adjuvant chemotherapy for early-stage breast cancer. J Clin Oncol 2005, , 25: 6027-36, Sep 1;23.

30. Rendell JM, Merritt RD, Geddes JR: Incentives and disincentives to participation by clinicians in randomised controlled trials. Cochrane Database Syst Rev 2007, , 2: MR000021, Apr 18.

31. Bell-Syer SE, Thorpe LN, Thomas K, et al: GP Participation and Recruitment of Patients to RCTs: Lessons from Trials of Acupuncture and Exercise for Low Back Pain in Primary Care. Evid Based Complement Alternat Med 2008.

32. Sikorskii A, Wyatt GK, Siddiqi AE, et al: Recruitment and Early Retention of Women with Advanced Breast Cancer in a Complementary and Alternative Medicine Trial. Evid Based Complement Alternat Med 2009.

33. Mao JJ, Farrar JT, Xie SX, Bowman MA, Armstrong K: Use of complementary and alternative medicine and prayer among a national sample of cancer survivors compared to other populations without cancer. Complement Ther Med 2007, , 1: 21-9, Mar;15 Epub 2006 Sep 28.

34. Vickers AJ, Ballen V, Scher H: Setting the bar in phase II trials: The use of historical data for determining "go/no go" decision for definitive phase III testing. Clin Cancer Res 2007, 13(3).

35. Buchanan DR, White JD, O'Mara AM, et al: Research-design issues in cancer-symptom-management trials using complementary and alternative medicine: lessons from the National Cancer Institute Community Clinical Oncology Program experience. J Clin Oncol 2005, 27: 6682-9, Sep 20;23.

36. Graves KD: Social cognitive theory and cancer patients' quality of life: a meta-analysis of psychosocial intervention components. Health Psychol 2003, , 2: 210-9, Mar;22.

37. Rogers LQ, Matevey $C$, Hopkins-Price $P$, et al: Exploring social cognitive theory constructs for promoting exercise among breast cancer patients. Cancer Nurs 2004, , 6: 462-73, Nov-Dec;27. 
38. Lee LL, Arthur A, Avis M: Using self-efficacy theory to develop interventions that help older people overcome psychological barriers to physical activity: a discussion paper. Int I Nurs Stud 2008, , 11: 1690-9, Nov;45.

39. van de Laar KE, van der Bijl Jj: Strategies enhancing self-efficacy in diabetes education: a review. Sch Inq Nurs Pract 2001, , 3: 235-48, Fall;15.

40. Allen NA: Social cognitive theory in diabetes exercise research: an integrative literature review. Diabetes Educ 2004, , 5: 805-19, Sep-Oct;30.

41. Boon H, Macpherson H, Fleishman S, et al: Evaluating Complex Healthcare Systems: A Critique of Four Approaches. Evid Based Complement Alternat Med 2007, , 3: 279-285, Sep;4.

42. Verhoef MJ, Vanderheyden LC, Fønnebø V: A whole systems research approach to cancer care: why do we need it and how do we get started? Integr Cancer Ther 2006, , 4: 287-92, Dec;5.

43. Ritenbaugh C, Hammerschlag R, Calabrese C, et al: A pilot whole systems clinical trial of traditional Chinese medicine and naturopathic medicine for the treatment of temporomandibular disorders. J Altern Complement Med 2008, 5: 475-87, Jun;14.

44. Jacobs $S R$, Jacobsen PB, Booth-Jones $\mathrm{M}$, et al: Evaluation of the functional assessment of cancer therapy cognitive scale with hematopoietic stem cell transplant patients. J Pain Symptom Manage 2007, , 1: 13-23, Jan;33.

45. Heritier S, Cantoni E, Copt S, et al: Robust Methods in Biostatistics. John Wiley and sons Ltd, West Sussex, United Kingdom; 2009.

46. Cannistra SA: Phase II trials in journal of clinical oncology. J Clin Oncol 2009, 27(19):3073-6

47. Rubenstein LV, Korn EL, Friedlin B, et al: Design issues of randomized phase II trials and a proposal for phase II screening trials. Journal of Clinical Oncology 2005, 23(28):7199-7206.

48. Revicki DA, Cella D, Hays RD, et al: Responsiveness and minimal important differences for patient reported outcomes. Health Qual Life Outcomes 2006, 4:70.

49. Haines T, Gross AR, Burnie $S$, et al: A Cochrane review of patient education for neck pain. Spine 2009, 9(10):859-71.

\section{Pre-publication history}

The pre-publication history for this paper can be accessed here: http://www.biomedcentral.com/1472-6882/11/49/prepub

doi:10.1186/1472-6882-11-49

Cite this article as: Johnston et al:: Patient education integrated with acupuncture for relief of cancer-related fatigue randomized controlled feasibility study. BMC Complementary and Alternative Medicine 2011 11:49.

\section{Submit your next manuscript to BioMed Central and take full advantage of:}

- Convenient online submission

- Thorough peer review

- No space constraints or color figure charges

- Immediate publication on acceptance

- Inclusion in PubMed, CAS, Scopus and Google Scholar

- Research which is freely available for redistribution

Submit your manuscript at www.biomedcentral.com/submit
Biomed Central 